\title{
An Artificial Robot Nervous System To Teach Robots How To Feel Pain And Reflexively React To Potentially Damaging Contacts
}

\author{
Johannes Kuehn and Sami Haddadin
}

\begin{abstract}
In this paper, we introduce the concept of an artificial Robot Nervous System (aRNS) as a novel way of unifying multi-modal physical stimuli sensation with robot painreflex movements. We focus on the formalization of robot pain, based on insights from human pain research, as an interpretation of tactile sensation. Specifically, pain signals are used to adapt the equilibrium position, stiffness, and feedforward torque of a pain-based impedance controller. The schemes are experimentally validated with the KUKA LWR4+ for simulated and real physical collisions using the BioTac ${ }^{\circledR}$ sensor.

Index Terms-Physical Human-Robot Interaction, Compliance and Impedance Control, Biologically-Inspired Robots, Biomimetics, Force and Tactile Sensing.
\end{abstract}

\section{INTRODUCTION}

$\mathbf{P}$ HYSICAL human-robot interaction (pHRI) has become an increasingly central discipline in robotics research. With regard to human safety, considerable research was carried out in [1], [2] and the results reached real-world applications and international standards. However, rather limited efforts were undertaken to ensure the robot's own safety via suitable controls and a systematic approach to do so is still missing. For this, a robot needs to be able to detect and classify unforeseen physical states and disturbances, rate the potential damage they may cause to it, and initiate appropriate countermeasures, i.e., reflexes. In turn, enhanced robot reflexes may improve human safety in case of human-robot collisions. In order to tackle this demanding requirement, the human antetype shall serve as our inspiration, meaning that human pain-reflex movements are used for designing according robot pain sensation models and reaction controls. For this, it is worthwhile to first take a closer look at human reflexes caused by pain - we denote painreflexes - since they are generally regarded of vital importance to the human.

\section{A. Human Nervous System \& Pain Reflexes}

Consisting of millions of connected neurons, the Human Nervous System (HNS) is the most powerful measurement and communication system of the human body. From a topological point of view the HNS can be divided into the Central and the Peripheral Nervous System (CNS, PNS). The CNS consists of the spinal cord, brain stem and forebrain and is mainly

Manuscript received August 31, 2015; revised December 19, 2015; accepted January 29, 2016.

This paper was recommended for publication by Editor Y. Yokokohji upon Associate Editor and Reviewers' comments. This work was supported by the European Union's Horizon 2020 research and innovation programme under Grant 688857.

Johannes Kuehn and Sami Haddadin are with Institute of Automatic Control (IRT), Faculty of Electrical Engineering and Computer Science, Leibniz Universität Hannover, Appelstr. 11 D-30167 Hannover, Germany kuehn, haddadineirt. uni-hannover. de

Digital Object Identifier (DOI): see top of this page. responsible for forwarding and processing information coming from the PNS [3]. The PNS covers all the nerves that are located outside the CNS, gathering tactile and proprioceptive information with the help of a wide spectrum of receptors. If a stimulus is that strong that body injuries become possible, a highly unpleasant, but vitally important, sensation is caused - pain [3]. The most responsible and contributing receptors to pain caused by physical contact are called nociceptors.

When a mechanical stimulus affects nociceptors, which are distributed all over the skin, they become activated instantly. Depending on the stimulus strength, typically in terms of duration, penetration depth and stress, the nociceptors send ionic electrical spikes decoded in a frequency modulated manner [3]. Once the stimulus surpasses a certain threshold, a nociceptor starts firing following the all-or-nothing principle at constant amplitude [3]. After the signal passes the first neural interconnection it crosses to the other side of the spinal cord, following the spinothalamic tract, then through medulla, pons and midbrain to the thalamus. From there on, pain information is projected to different areas of the cerebral cortex. Pain is evoked, which may cause appropriate movements (pain-reflexes). Obviously, pain is also strongly an emotional experience, not only influenced by the signals coming from the nociceptors. Thus, one distinguishes between the emotional experience of pain and the nociceptive signals that may lead to pain experiences. In this paper, we focus on the latter.

\section{B. Reflex Control in Robotics}

Various approaches to reflexes in legged robot locomotion were developed with focus on stabilizing the gait of humanoids in [4], [5], [6]. Reflexes in manipulation tasks differ, since they are less cyclic and rather asymmetric. In [7], the humanoid "Cog" was taught reflexive behaviors by generating movements from biologically predefined inspired postural primitives. Through the superposition of these primitives it was possible to imitate reflex-like withdrawal behavior. In [8], a similar approach was used by overlaying different movement patterns triggered by a tactile (force) sensor and was implemented on a 7-Degree-of-Freedom (DoF) robot arm. In [9], withdrawal movements of the human-arm were recorded and a force-distance relation was established to model this behavior. In order to trigger reflexes, an elastic robot-tissue was introduced, additionally mitigating the impact force through inherent damping properties. Moreover, in [10], based on the work in [11], [12], various collision reflex controls were proposed in the framework of proprioceptive collision detection and reflex reaction. Further approaches to reflex control based on optimal control can be found in [13] and [14]. 
Early reflex control approaches are mostly based on single sensors, e.g., via force-sensors that trigger predefined patterns. In [8], the authors activate reflexes by modeling single neuron firing per reflex-type. However, they focus on manipulation and specific rather abstract high-level reflexes such as grasping or catching in case of slipping. The basic implementation of mechanically triggered reflexes is also based on force sensing only. To the best of the authors' knowledge, no deeper "mechano-physiological" contact dynamics were involved, nor does their human-like neuron firing imply human topological design. It rather represents a switching strategy that may trigger different events.

\section{Contribution}

In this paper, we introduce the artificial Robot Nervous System aRNS as a new way of integrating tactile sensation and according reflex reactions into robot control based on the concept of robot pain sensation. During a mechanical stimulus, the penetration stress, depth, and its dynamics are sensed via a virtual nervous robot-tissue and corresponding pain spiking signals are generated. Together with an interpretation of pain level, the signals are then used to activate suitable pain-reflex movements. Concretely, the proposed pain-based controller serves, e.g., for online adaptation of the equilibrium position, stiffness, and feed-forward torque of a joint-level pain-based impedance controller. The overall behavior allows the robot to sensitively interact with its environment at nominal pain level, while mitigating potential risks by activating human inspired reflex strategies if the pain level increases. Note that the proposed framework can analogously be extended to other modalities.

The paper is organized as follows. In Section II the design of the aRNS is introduced. We explain the underlying concept, the required definitions, and the proposed mathematical model. Furthermore, the signal interpretation and corresponding pain reaction strategies are discussed. The pain-based joint-level impedance controller is introduced in Sec. III. Section IV reports on the results for simulated and real physical contact events using the BioTac sensor. The sensor is mounted on the robot's end effector and utilized as a real world implementation of the proposed sensory model. Finally, we conclude the paper in Sec. V.

\section{Artificial Robot Nervous System}

\section{A. Concept}

In general, the schematic concept of a collision between any suitably controlled (via the desired torque $\tau_{d}$ ) robot and a collision object (mass $m_{c}$, local radius $r_{c}$ ) can be described by its corresponding contact dynamics, see Fig. 1. In generalized coordinates, the contact dynamics between a robot arm and a colliding object with state $\boldsymbol{x}_{c}$ and its derivative $\dot{\boldsymbol{x}}_{c}$ are determined by the robot joint configuration $\boldsymbol{q}$, the joint velocity $\dot{\boldsymbol{q}}$, and the external torque vector $\tau_{\text {ext }}$. This torque is caused by the contact wrench $\mathcal{F}_{c}$ or the stress $\sigma_{c}$ (single point contact) acting on the collision object and the robot, respectively. In order to rate potentially painful collisions and activate proper pain-reflexes, using information about $\tau_{\text {ext }}$ only may not be sufficient. Humans are known to utilize further information, e.g., in terms of penetration depth $\delta$ or stress, into their controls, using the multitude of information provided by the skin; and not only from their proprioceptive torque measurement via

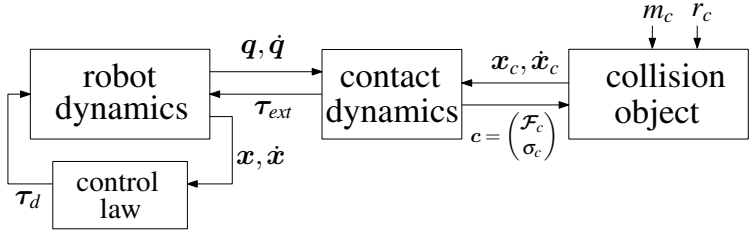

Fig. 1. Dynamics of a collision between a controlled robot and a collision object.

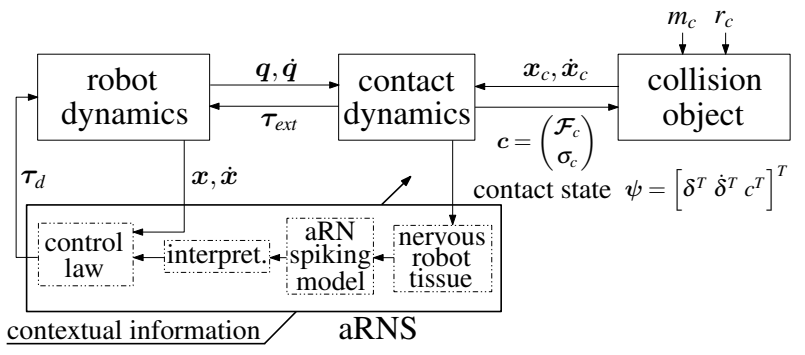

Fig. 2. Collision between a controlled robot and a collision object including an artificial Robot Nervous System (aRNS).

the golgi tendon organs. In this paper, our goal is to enhance robots with similar capabilities by introducing the concept of an artificial Robot Nervous System that is inspired by the HNS. This enables a robot to not only sense, but also to interpret and react to "painful" collisions, see Fig. 2. The aRNS can be subdivided into four major components: the nervous robottissue, the spiking model, the interpretation layer of generated spiking signals, and the motor control law. In the following, each component is separately elucidated, while the proposed control laws are introduced in Sec. III.

\section{B. Mechanical Model of the Virtual Nervous Robot-Tissue}

We assume a hypothetical nervous robot-tissue model that is inspired by the human skin structure. In analogy to the human skin, we split the nervous robot-tissue into three distinct layers filled with artificial Robot Neurons (aRNs) that replicate the principles of human receptors, see Fig. 3. The parameters $b_{1,2,3}$ denote the thickness of each layer and are set to $b_{1}=0.002 \mathrm{~m}, b_{2}=0.004 \mathrm{~m}$, and $b_{3}=0.014 \mathrm{~m}$, resulting in a total tissue thickness $b_{t}=0.02 \mathrm{~m}$. The contact with an object is described by the contact radius $d$, the penetration volume $V_{c}$, and the object radius $r_{c}$. The material constants $E_{1,2,3}$ and $v_{1,2,3}$ represent the elastic modules and the Poisson ratios. In this paper, they are chosen to be $E_{1}=E_{2}=E_{3}:=50 \mathrm{MPa}$ and $v_{1}=v_{2}=v_{3}:=0.25 . E_{1,2,3}$ and $v_{1,2,3}$ correspond to polystyrene (soft) material that may be a suitable choice for a mechanical implementation. Based on [15], $b_{t}$ is chosen to be thick enough to prevent the material from full compression during contact events. $b_{1,2,3}$ are chosen in terms of a scaled ratio that approximately corresponds to the standard thickness ratio of human skin layers [3]. The parameters $\rho_{1,2,3}$ represent the homogenous density of the aRNs and were chosen to be $\rho_{1}<\rho_{2}<\rho_{3}$ with $\rho_{1}=10^{7} \mathrm{~m}^{-3}, \rho_{2}=1.05 \cdot 10^{7} \mathrm{~m}^{-3}$, and $\rho_{3}=1.1 \cdot 10^{7} \mathrm{~m}^{-3}$. These density values approximately correspond to the density of the human fingertip mechanoreceptors [16]. ${ }^{1}$ This contact structure has two main properties. First, the deeper a collision object penetrates into the nervous robottissue the more aRNs are stimulated. Second, when a collision

\footnotetext{
${ }^{1}$ For more details on suitable contact models, please refer to [15].
} 


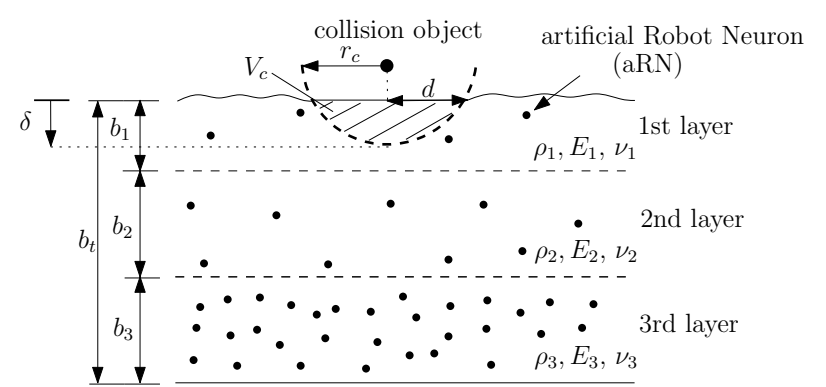

Fig. 3. Mechanical model of the proposed nervous robot-tissue.

object reaches the next tissue layer the rate of stimulated aRNs rises. Note that the distribution of aRNs does not necessarily reflect the distribution of human skin receptors.

\section{Spiking Models}

1) Principle aRN spiking characteristics: Basically, the aRNs reflect the basic operation of human receptors. If the stimulus of human neurons exceeds a certain threshold, they start to send spike-like signals. One spike is constant at amplitude, duration and refractory time, which denotes the duration the receptor is not able to fire again regardless of present stimuli. A significant amount of information is thus encoded by changing the frequency of the firing rate. To imitate such behavior, aRN firing can be modeled as a finitestate machine (FSM) consisting of the three states idle-, firingand rest-state, see Fig. 4. Initially, the aRN is in idle-state, i.e.,

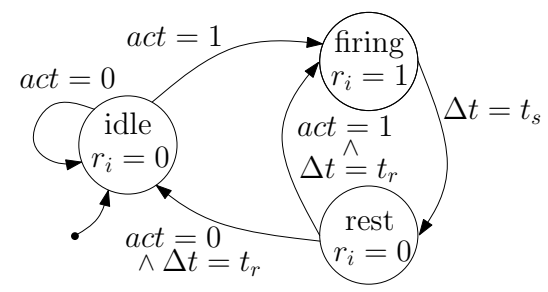

Fig. 4. Modeling aRN firing as a finite-state machine.

in case of no contact ( $a c t=0$ ) leading to $r_{i}=0$. Subscript $i \in \mathbb{N}$ denotes the individual aRN. If stimulation occurs $(a c t=1)$ the aRN switches to the firing-state, i.e., it starts firing by setting $r_{i}=1$ for a fixed time duration $t_{s}$. Thereafter, it switches to rest-state. The output is then set to $r_{i}=0$ for the refractory time $t_{r}$, regardless of possibly present stimuli. After this silent period, the aRN switches either back to firing-state $(a c t=1)$ or remains silent by switching to idle-state $(a c t=0)$, depending on the stimulus. In the following, the aRN types modeled in this paper are outlined.

2) aRN types: Here, aRN types that respond to penetration depth, penetration velocity and compressive stress, including their spiking models, are elaborated as they correlate with pain sensation as described in [17], [18]. ${ }^{2}$ We assume each aRN type to be equally distributed over the robot-tissue with according density $\rho_{1,2,3}$, respectively. In addition to the "instantaneous" aRN types we also introduce aRNs encoding the repetitiveness of contacts, since it is known that this influences pain level as well [19].

\footnotetext{
${ }^{2}$ In this paper, we add penetration velocity, which obviously strongly correlates with potential danger, as an important metric for an aRNS. We anticipate that such a modality will become technologically available in the future.
}

a) Penetration depth spike train: Under the assumption that the higher the penetration depth $\delta$, the more aRNs are activated, the total number of activated aRNs $r(\boldsymbol{\delta})$ is

$$
r(\delta)= \begin{cases}V_{1}(\delta) \rho_{1} & \delta \leq b_{1} \\ V_{2}\left(\delta-b_{1}\right) \rho_{2}+r\left(b_{1}\right) & b_{1}<\delta \leq b_{t 2} \\ V_{3}\left(\delta-b_{t 2}\right) \rho_{3}+r\left(b_{t 2}\right) & b_{t 2}<\delta,\end{cases}
$$

with $b_{t 2}=b_{1}+b_{2} . V_{1}(\boldsymbol{\delta}), V_{2}\left(\boldsymbol{\delta}-b_{1}\right)$ and $V_{3}\left(\boldsymbol{\delta}-b_{t 2}\right)$ denote the instantaneous effective volumes that envelop the stimulated aRNs. Due to tissue compression, those aRNs that were placed at penetration volume $V_{c}$ (see Fig. 3) push against neighboring ones and may stimulate them as well. Therefore, some of the affected aRNs lie outside the penetration volume $V_{c}$. To take this effect into account, we approximate the instantaneous effective volumes to be cylindrical as a reasonable simplification. Accordingly, each respective volume of layer $i$ is obtained by

$$
V_{i}(\delta)=\Delta \delta_{i} \pi d^{2}=\Delta \delta_{i} \pi\left(2 r_{c} \Delta \delta_{i}-\Delta \delta_{i}^{2}\right),
$$

where $\Delta \delta_{i}$ is the penetration depth in layer $i$ and $d$ the radius of the cylindric volume (see Fig. 3).

b) Penetration velocity spike train: The velocitydependent spike generation is defined in a straight forward manner as the absolute value of the first time derivative of penetration depth based firing

$$
r_{v}:=|\dot{r}(\delta)| .
$$

c) Compressive stress spike train: The stress-dependent spiking is computed as

$$
p(\sigma)= \begin{cases}V_{1}\left(\delta^{*}\right) \rho_{1} & \delta^{*} \leq b_{1} \\ V_{2}\left(\delta^{*}-b_{1}\right) \rho_{2}+r\left(b_{1}\right) & b_{1}<\delta^{*} \leq b_{t 2} \\ V_{2}\left(\delta^{*}-b_{t 2}\right) \rho_{3}+r\left(b_{t 2}\right) & b_{t 2}<\delta^{*}\end{cases}
$$

Due to the physically decaying impact characteristics of pressure we introduce the effective penetration depth $\delta^{*}$ as

$$
\delta^{*}:= \begin{cases}c_{e} \delta & \delta \leq \frac{b_{t}}{2} \\ b_{t} & \frac{b_{t}}{2}<\delta \leq b_{t},\end{cases}
$$

since not only the aRNs within the penetration depth $\delta$ are activated, but also significant portions below. We chose $c_{e} \approx 2$, then, as a result $\delta^{*}$ covers $99 \%$ of the impact decay, i.e., the tissue stress has reduced to $<1 \%$ of the maximum surface stress. In analogy to (2) the instantaneous stress volumes are

$$
V_{i}\left(\delta^{*}\right)=\Delta \delta_{i}^{*} \pi d^{2}, \quad i=1,2,3,
$$

where $\Delta \delta_{i}^{*}$ is the effective penetration depth of layer $i$.

d) Repetitiveness spike train: The repetitiveness $\xi$ is defined as

$$
\xi:=\int_{t_{c}} \dot{\xi} \mathrm{d} t,
$$

where $t_{c}$ is the time instant at which the collision occurs. The repetitveness rate $\dot{\xi}$ is defined as

$$
\dot{\xi}:= \begin{cases}a F(\delta) \dot{\delta} & \dot{\delta}>0 \\ -\int_{t_{v}} \ddot{\xi}_{r} \mathrm{~d} t & \dot{\delta} \leq 0 \wedge \xi>0 \\ 0 & \dot{\delta} \leq 0 \wedge \xi=0\end{cases}
$$

where $a>0$ and $\ddot{\xi}_{r}>0$ are the growth and decay factors. $t_{v}$ denotes the time when penetration velocity gets negative. The contact force $F(\delta)$ is assumed to follow Hertzian theory, see [15].

Figure 5 depicts the sensory response of the nociceptive aRNs given the true compressive stress $\sigma$ under the following 
collision parameters. The contact occurs between a 2-shell 1-DoF robot with $m_{r}=4.5 \mathrm{~kg}, r_{r}=0.1 \mathrm{~m}$ and a 1-shell collision object with $m_{c}=4.5 \mathrm{~kg}, r_{c}=0.1 \mathrm{~m}$ at impact speed $v_{c}=0.4 \mathrm{~m} / \mathrm{s}$. The robot is assumed to be at rest and the object approaches at $v_{c}$. After a single contact event a repetitive decaying impact follows. The response of the respective aRNtype to penetration depth $r(\delta)$, velocity $r_{v}$, impact stress $p(\sigma)$ and repetition frequency $\xi$ are displayed. One can see that each $\mathrm{aRN}$ type correlates with the respective (physical) modality.

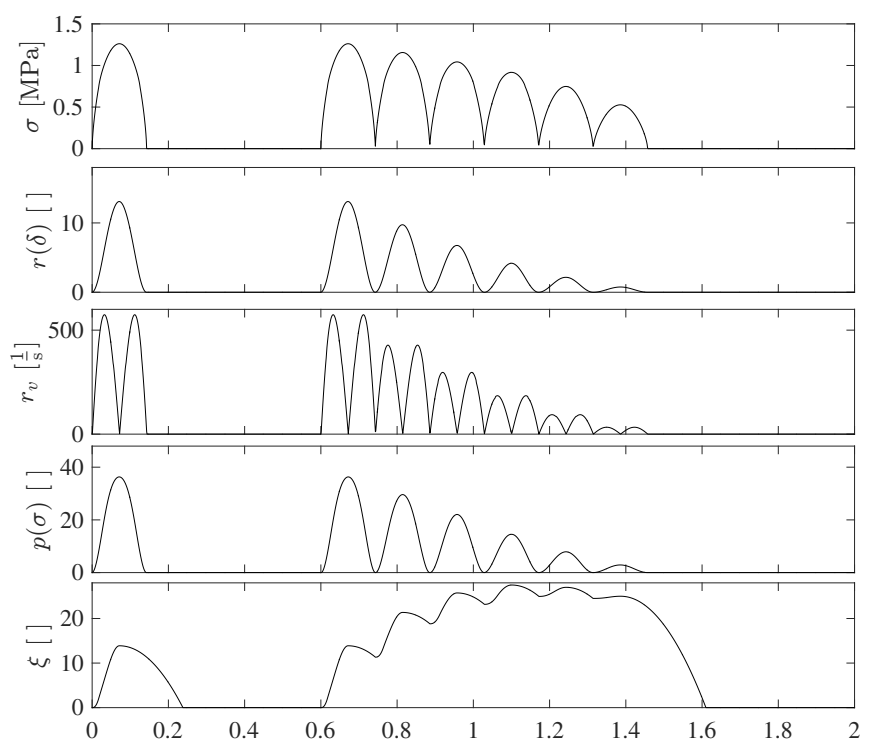

Fig. 5. Cumulated spike signals of aRN-types penetration depth $r$, velocity $r_{v}$, stress $p$, and repetitiveness $\xi$.

3) Implementation remarks: For convenience, we introduce a time discretisation $t=k T_{s}$, with sampling time $T_{s}$ and time step $k \in \mathbb{N}$, in the implementation. During the refractory time $t_{r}$ (see Fig. 4) aRNs do not fire. Thus, the number of stimulated aRNs $r\left(\delta, k T_{s}\right)$ in (1) reduces to an effective number $r_{e f f}$. This is the sum of aRNs currently being in firing-state. By setting $t_{r}=t_{s}=T_{s}$ the stimulated aRNs can be either in firing or silent mode for every $k$. Consider the stimulated aRNs at time step $k$ fire. Then they pause in time step $k+1$ and fire again in $k+2$ (assuming the stimulus is still present, i.e., act $=1$, see Fig. 4). The icrement of stimulated aRNs in time step $k$ is therefore

$$
\Delta r_{i}\left(\delta, k T_{s}\right)=r\left(\delta, k T_{s}\right)-r\left(\delta,(k-1) T_{s}\right), \quad i=1,2, \ldots, l,
$$

where subscript $i$ is the incremental counter during contact. All $\Delta r_{i}\left(\delta, k T_{S}\right)$ form the elements of the stacked vector

$$
\Delta R=\left[\begin{array}{lll}
\Delta r_{1} & \ldots & \Delta r_{l}
\end{array}\right]^{T} \quad \Delta R \in \mathbb{R}^{l}, \quad \Delta r_{i}>0
$$

of length $l$ that increases as long as $\Delta r_{i}>0$. It follows that for even and odd time steps one can separately compute $r_{e f f}$ as

$$
r_{e f f}\left(k T_{s}\right)=\left\{\begin{array}{ll}
\sum_{j=0}^{\lfloor l / 2\rfloor} \Delta R_{2 j+1} & k=2 m+1 \\
\sum_{j=1}^{\lfloor l / 2\rfloor} \Delta R_{2 j} & k=2 m
\end{array} \quad, m=0,1,2, \ldots .\right.
$$

If $\Delta r_{i}$ is negative $\left(\Delta r_{i}^{-}:=\Delta r_{i} \leq 0\right)$ the number of stimulated aRNs decreases. This means that the object's collision velocity turns into the opposite direction and thus moves away from the robot. Therefore, $\Delta r_{i}^{-}$is deleted from $\Delta R$ in reverse order such that the aRNs stimulated last are deactivated first. Algorithm 1 shows the pseudo code of this firing reduction algorithm.

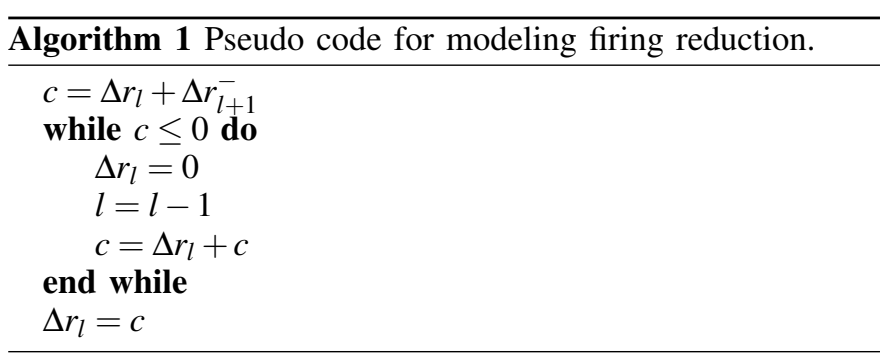

\section{Interpretation}

In this paper, robot pain is defined as the interpretation of spike trains generated by the aRNs involving contextual information. Inspired by the human pain system, robot pain is divided into four verbal pain classes: no, light, moderate, and severe pain. They represent a simplification of the Verbal Rating Scale (VRS) of pain measurement in humans, see Tab. I. The first pain class contains no contact as well as contacts that are not painful, we call those soft contacts. Consider, e.g., the robot fulfills a certain task such as holding a desired configuration $\boldsymbol{q}_{d}$. Obviously, in case of no contact the robot shall hold its position. In case of soft contact, the robot experiences an external torque $\tau_{\text {ext }}$ that results in a deviation from its desired equilibrium position $\boldsymbol{q}_{d}$. Since the contact is not harmful the robot shall treat the contact as a disturbance, compensate for it, and focus on the desired task. In the second pain class, such contacts occur that may harm the robot or prevent it from performing the task. The robot "feels" uncomfortable and shall smoothly retract until the contact event is over and return thereafter. Strong collisions are covered in the third pain class. The robot "feels" moderate pain, shall quickly retract, and more distant until the contact event is over. Then, it may move back towards $\boldsymbol{q}_{d}$. The last pain class covers all contacts in which the robot may be damaged and thus needs some sort of "help". In order to prevent making the damage worse, the robot switches to gravity compensation with additional damping for dissipation, improving the safety of the robot and the environment by its strictly passive behavior. The desired interpretation is realized by the control laws that are elucidated in the following.

\section{ARNS: COLLISION CONTROL}

\section{A. Robot dynamics}

For the pain-based controller design, we assume an $n$-DoF serial chain rigid robot to be equipped with the aRNS. The dynamics of such a system in contact can be described in Lagrangian form by

$$
\boldsymbol{\tau}=\boldsymbol{M}(\boldsymbol{q}) \ddot{\boldsymbol{q}}+\boldsymbol{C}(\boldsymbol{q}, \dot{\boldsymbol{q}}) \dot{\boldsymbol{q}}+\boldsymbol{g}(\boldsymbol{q})+\boldsymbol{\tau}_{\mathrm{ext}},
$$

where $\boldsymbol{\tau} \in \mathbb{R}^{n}$ denotes the actuator torques, $\boldsymbol{M}(\boldsymbol{q}) \in \mathbb{R}^{n \times n}$ is the symmetric joint inertia matrix, $\boldsymbol{C}(\boldsymbol{q}, \dot{\boldsymbol{q}}) \in \mathbb{R}^{n \times n}$ the Coriolis and centrifugal matrix, $\boldsymbol{g}(\boldsymbol{q}) \in \mathbb{R}^{n}$ the gravity vector, and $\tau_{\text {ext }}$ the external joint torques. Let us consider a single-areal contact with associated contact Jacobian matrix $\boldsymbol{J}_{c}(\boldsymbol{q})$. This maps the physically acting contact wrench $\mathcal{F}_{\text {ext }} \in \mathbb{R}^{6}$, which is caused by the local compression and tensile stress distribution, into the respective external joint torques via

$$
\boldsymbol{\tau}_{\text {ext }}=\boldsymbol{J}_{c}^{T}(\boldsymbol{q}) \mathcal{F}_{\text {ext }}=\boldsymbol{J}_{c}^{T}(\boldsymbol{q})\left[\boldsymbol{f}_{\text {ext }} \boldsymbol{m}_{\text {ext }}\right]^{T}
$$


TABLE I

ROBOT PAIN CLASSES AND CORRESPONDING REACTION STRATEGIES.

\begin{tabular}{lllllll}
\hline Robot pain class & Collision severity & Strategy & $\boldsymbol{q}_{d}$-adapt. & $\boldsymbol{K}_{d}$-adapt. & $\boldsymbol{\tau}_{f f}$-adapt. \\
\hline I & no pain & no/soft contact & fulfill task/compensate & $(16)$, with $\dot{\boldsymbol{q}}_{p}\left(\boldsymbol{k}_{\boldsymbol{q}}^{I}\right)$ & $(19)$, with $\boldsymbol{K}_{d}\left({ }^{I} \boldsymbol{K}_{d}^{*}, \boldsymbol{k}_{\boldsymbol{K}}^{I}\right)$ & $(21)$, with $\boldsymbol{\tau}_{f f}\left(\boldsymbol{k}_{\boldsymbol{\tau}}^{I}, \boldsymbol{W}>\operatorname{diag}(\mathbf{0})\right)$ \\
II & light pain & light & smoothly retract & $(16)$, with $\dot{\boldsymbol{q}}_{p}\left(\boldsymbol{k}_{\boldsymbol{q}}^{I I}\right)$ & (19), with $\boldsymbol{K}_{d}\left({ }^{I I} \boldsymbol{K}_{d}^{*}, \boldsymbol{k}_{\boldsymbol{K}}^{I I}\right)$ & $(21)$, with $\boldsymbol{\tau}_{f f}\left(\boldsymbol{k}_{\boldsymbol{\tau}}^{I I}, \boldsymbol{W}=\operatorname{diag}(\mathbf{0})\right)$ \\
III & moderate pain & strong & quickly retract & (16), with $\dot{\boldsymbol{q}}_{p}\left(\boldsymbol{k}_{\boldsymbol{q}}^{I I I}\right)$ & (19), with $\boldsymbol{K}_{d}\left({ }^{I I I} \boldsymbol{K}_{d}^{*}, \boldsymbol{k}_{\boldsymbol{K}}^{I I I}\right)$ & $(21)$, with $\boldsymbol{\tau}_{f f}\left(\boldsymbol{k}_{\boldsymbol{\tau}}^{I I I}, \boldsymbol{W}=\operatorname{diag}(\mathbf{0})\right)$ \\
IV & severe pain & hard & abort task & n.a. & n.a. & n.a. \\
\hline
\end{tabular}

In this paper, we assume the unit collision direction $\boldsymbol{u}_{c}$ of $\mathcal{F}_{\text {ext }}$ and $\boldsymbol{J}_{c}$ to be known. In general this information could be derived from various sources such as e.g. proprioceptive information or suitable tactile sensors.

\section{B. Generalized Pain Spiking State}

In order to involve the spiking signals into the overall control strategy, we introduce the generalized pain spiking state as

$$
s(t)=\left[p(\sigma) r(\delta) r_{v}(\delta) \xi\right]^{T} \geq \mathbf{0},
$$

which is defined as the stacked vector of all cumulated spike signals from Sec. II-C2. Note, for a possibly real world implementation of the aRNS using conventional sensors, i.e. providing analogue outputs, one can use this spiking state vector as an entry point to incorporate those signals. For example, in this paper, we subsequently use the BioTac analogue outputs to replace the cumulated spike signals, leading to a sensor related spiking vector $s_{B T}$, see Sec. IV-B.

\section{Reflex Control Strategies}

The control strategy we propose adapts the overall impedance and feed-forward characteristics of a joint level impedance controller, as well as the reference trajectory based on the Cartesian pain sensation and interpretation. This painbased joint level impedance controller is defined by

$$
\boldsymbol{\tau}_{d}=\boldsymbol{K}_{d}(\boldsymbol{s})\left(\boldsymbol{q}_{d}(\boldsymbol{s})-\boldsymbol{q}\right)+\boldsymbol{D}(\boldsymbol{q}) \dot{\boldsymbol{q}}+\boldsymbol{\tau}_{f f}(\boldsymbol{s})+\boldsymbol{\tau}_{G}(\boldsymbol{q}),
$$

where $\boldsymbol{K}_{d} \in \mathbb{R}^{n \times n}, \boldsymbol{q}_{d} \in \mathbb{R}^{n}$ and $\boldsymbol{\tau}_{f f} \in \mathbb{R}^{n}$ are the desired closed loop stiffness, the desired equilibrium position and the feedforward torque. They all depend on the generalized pain spiking state $s$. The vector $\tau_{G} \in \mathbb{R}^{n}$ denotes the gravity compensation torque and $D \in \mathbb{R}^{n \times n}$ the configuration dependent joint damping matrix

$$
\boldsymbol{D}(\boldsymbol{q})=\boldsymbol{A}(\boldsymbol{q}) \boldsymbol{D}_{\xi} \boldsymbol{K}_{d 1}+\boldsymbol{K}_{d 1} \boldsymbol{D}_{\xi} \boldsymbol{A}(\boldsymbol{q}),
$$

where $\boldsymbol{D}_{\xi}=\operatorname{diag}\left(\frac{1}{\sqrt{2}}\right) \in \mathbb{R}^{n \times n}$ is the desired joint damping ratio matrix. According to [20], $\boldsymbol{A}(\boldsymbol{q})$ and $\boldsymbol{K}_{d 1}$ are defined by $\boldsymbol{A}(\boldsymbol{q}) \boldsymbol{A}(\boldsymbol{q})=\boldsymbol{M}(\boldsymbol{q})$ and $\boldsymbol{K}_{d 1} \boldsymbol{K}_{d 1}=\boldsymbol{K}_{d}$.

a) $\boldsymbol{q}_{d}$-adaptation: Equilibrium position adaptation after the collision event, occurring at collision time $t_{c}$, shall cause faster evading from external contacts the larger $s(t)$. After the contact, an exponential recovery behavior towards the original equilibrium $\boldsymbol{q}_{d}\left(t_{c}\right)$ shall be achieved. Specifically, the desired equilibrium position $\boldsymbol{q}_{d}$ is adapted via

$$
\boldsymbol{q}_{d}:=\boldsymbol{q}_{d}\left(t_{c}\right)+\int_{t_{c}} \dot{\boldsymbol{q}}_{p}(\boldsymbol{s}) \mathrm{d} t
$$

where $\boldsymbol{q}_{d}\left(t_{c}\right)$ denotes the desired joint configuration at $t_{c}$ and $\dot{\boldsymbol{q}}_{p}$ is the pain reflex equilibrium rate, which is integrated from collision time $t_{c}$ on. The generalized virtual pain force $\boldsymbol{f}_{v}$ is defined as

$$
\boldsymbol{f}_{v}:=\left(\boldsymbol{k}_{\boldsymbol{q}}^{T} \boldsymbol{s}\right) \boldsymbol{u}_{c}
$$

where $\boldsymbol{k}_{\boldsymbol{q}}>\mathbf{0}$ is a gain vector. The pain reflex equilibrium rate is then obtained by

$$
\dot{\boldsymbol{q}}_{p}:= \begin{cases}-\boldsymbol{J}_{c}^{T} \mathcal{F}_{v}:=-\boldsymbol{J}_{c}^{T}\left[\boldsymbol{f}_{v} \mathbf{0}\right]^{T} & \boldsymbol{s}>\mathbf{0} \\ -\operatorname{sign}\left\{\boldsymbol{q}_{d}-\boldsymbol{q}_{d}\left(t_{c}\right)\right\} \int_{t^{\prime}} \ddot{\boldsymbol{q}}_{r} \mathrm{~d} t & \boldsymbol{s}=\mathbf{0} \wedge \boldsymbol{q}_{d} \neq \boldsymbol{q}_{d}\left(t_{c}\right) \\ \mathbf{0} & \boldsymbol{s}=\mathbf{0} \wedge \boldsymbol{q}_{d}=\boldsymbol{q}_{d}\left(t_{c}\right),\end{cases}
$$

where $\ddot{\boldsymbol{q}}_{r}>\mathbf{0}$ is the constant equilibrium recovery acceleration. $t^{\prime}$ is the time at contact loss.

b) $\boldsymbol{K}_{d}$-adaption: The stronger the stimulus, stiffness adaptation shall cause faster stiffness increase, while after the collision recovery to the original constant reference stiffness $\boldsymbol{K}_{d}^{*}>\mathbf{0}$ shall be moderately fast. For the sake of simplicity, we choose the stiffness entries to be equal for all joints. The desired diagonal closed loop stiffness $\boldsymbol{K}_{d}>\mathbf{0}$ is adapted by

$$
\boldsymbol{K}_{d}:=\boldsymbol{K}_{d}^{*}+\min \left[\Delta \boldsymbol{K}_{d, \max }, \int_{t_{c}} \dot{\boldsymbol{K}}_{p}(s) \mathrm{d} t\right],
$$

where $\dot{\boldsymbol{K}}_{p}$ is the pain stiffness rate. The stiffness increase (as reasoned in Sec. III-D), which is bounded from above by $\Delta \boldsymbol{K}_{d, \text { max }}=\boldsymbol{K}_{d, \text { max }}-\boldsymbol{K}_{d}^{*}$, starts at $t_{c}$. The according stiffness adaptation rate $\dot{\boldsymbol{K}}_{p}(s)$ is defined as

$$
\dot{\boldsymbol{K}}_{p}(s):= \begin{cases}\operatorname{diag}\left(\boldsymbol{k}_{\boldsymbol{K}}^{T} \boldsymbol{s}\right) & \boldsymbol{s}>\mathbf{0} \\ -\int_{t^{\prime}} \ddot{\boldsymbol{K}}_{\mathrm{r}} \mathrm{d} t & s=\mathbf{0} \wedge \boldsymbol{K}_{d}>\boldsymbol{K}_{d}^{*} \\ \mathbf{0} & \boldsymbol{s}=\mathbf{0} \wedge \boldsymbol{K}_{d}=\boldsymbol{K}_{d}^{*},\end{cases}
$$

where $\boldsymbol{k}_{K}>\mathbf{0}$ represents the stiffness rate gain vector and $\ddot{\boldsymbol{K}}_{\mathrm{r}}>\mathbf{0}$ the constant stiffness relaxation acceleration.

c) $\boldsymbol{\tau}_{f f}$-adaptation: The feed forward torque $\boldsymbol{\tau}_{f f}$ is adapted according to the same principle as the equilibrium position behavior. It consists of the pain reflex torque $\tau_{p}$ and the compensation torque $\tau_{\text {comp }}$ :

$$
\boldsymbol{\tau}_{f f}(\boldsymbol{s}):=\boldsymbol{\tau}_{p}(\boldsymbol{s})+\boldsymbol{\tau}_{\text {comp }}
$$

Let us start at the generalized pain force $\boldsymbol{f}_{p}$, which is defined as

$$
\boldsymbol{f}_{p}:=\left(\boldsymbol{k}_{\boldsymbol{\tau}}^{T} \boldsymbol{s}\right) \boldsymbol{u}_{c},
$$

where $\boldsymbol{k}_{\boldsymbol{\tau}}>\mathbf{0}$ is the gain vector of the pain reflex torque. The pain reflex torque is then obtained via

$$
\boldsymbol{\tau}_{p}(\boldsymbol{s}):= \begin{cases}-\boldsymbol{J}_{c}^{T} \mathcal{F}_{p}:=\boldsymbol{J}_{c}^{T}\left[\boldsymbol{f}_{p} \mathbf{0}\right]^{T} & \boldsymbol{s}>\mathbf{0} \\ -\operatorname{sign}\left\{\boldsymbol{\tau}_{p}\right\} \iint_{t^{\prime}} \ddot{\boldsymbol{\tau}}_{\mathrm{r}} \mathrm{d} t & \boldsymbol{s}=\mathbf{0} \wedge \boldsymbol{\tau}_{p} \neq \mathbf{0} \\ \mathbf{0} & \boldsymbol{s}=\mathbf{0}\end{cases}
$$


where $\mathcal{F}_{p}$ denotes the pain wrench and $\ddot{\tau}_{r}>\mathbf{0}$ the constant pain torque recovery acceleration. The compensation torque is computed as

$$
\boldsymbol{\tau}_{\text {comp }}:=\boldsymbol{W} \int_{t_{c}}\left(\boldsymbol{q}-\boldsymbol{q}\left(t_{c}\right)\right) \mathrm{d} t,
$$

where $\boldsymbol{W}>\mathbf{0} \in \mathbb{R}^{n \times n}$ represents a diagonal gain matrix. Note that the compensation torque does not depend on the spiking state vector $\boldsymbol{s}$. It is only active in the first pain class in order to compensate unintended (and painless) contacts.

\section{Pain Reflex Graph}

Figure 6 illustrates the pain reflex graph. $s_{1}, s_{2}$ and $s_{3} \in$

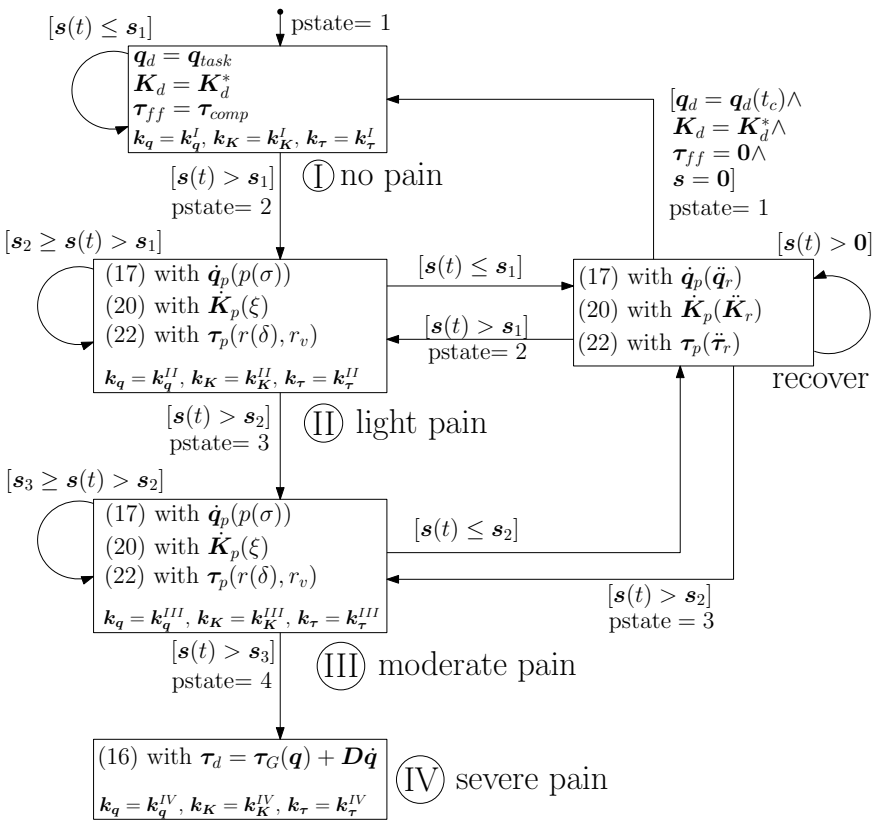

Fig. 6. Pain reflex graph.

$\mathbb{R}^{4 \times 1}\left(s_{1}<s_{2}<s_{3}\right)$ are the vectors containing the thresholds for transitioning between pain states. Note that the comparison with $s$ takes place element-wise. For evaluating when to switch to the next pain-state the compressive stress spiking $p(\sigma)$ is used. The variable pstate denotes the previously active pain state. In case of multiple contacts, the robot returns to the nominal pain class only after it has fully recovered from the previous pain level. Controls do not switch from higher to lower pain level without completely recovering to the nominal pain level first. Most noticeably, the equilibrium adaption $\boldsymbol{q}_{d}$ is set to correlate with the compressive stress that varies with the collision object shape. The stiffness adaption $\boldsymbol{K}_{d}$ is connected to repetitiveness in order to be abel to react to collision bursts faster. The feedforward torque adaption $\boldsymbol{\tau}_{f f}$ changes with penetration depth and velocity aRN spiking as a response to potentially painful collisions.

One can conclude, the higher the compressive stress, the more distant the equilibrium point is set. The higher the number of contact repetitions, the stiffer the controls. The deeper and faster the collision object penetrates, the stronger the feedforward torque. As the respectively growing intensities increase with the order of pain class according to the gain vectors, the decaying rates decrease, i.e., the time period of recovering grows with order of pain class. In pain class IV, controls are set to gravity compensation with a constant diagonal damping matrix $\boldsymbol{D}>\mathbf{0}$ only. Since $\boldsymbol{f}_{p}$ in (22) depends on two aRN types, the respective gain constants $k_{\tau, 2}$ and $k_{\tau, 3}$ are set such that they equally contribute to the maximally possible pain force $\boldsymbol{f}_{p, \max }$. Thus, the torque gain vector becomes

$$
\boldsymbol{k}_{\boldsymbol{\tau}}=\left[\begin{array}{c}
0 \\
\left(2 r_{\max }(\boldsymbol{\delta})\right)^{-1} \boldsymbol{f}_{p, \max } \\
\left(2 r_{v, \max }(\boldsymbol{\delta})\right)^{-1} \boldsymbol{f}_{p, \max } \\
0
\end{array}\right],
$$

where $r_{\max }(\delta)$ and $r_{v, \max }(\delta)$ denote the respective maximum spiking magnitude.

\section{EXPERIMENTAL RESULTS}

\section{A. Parameter dependency of aRNS control laws}

The parameter dependency of aRNS control laws is investigated during simulated single and multiple collision event(s) between a 2-shell 1-DoF robot $\left(m_{r}=4.5 \mathrm{~kg}, r_{r}=0.1 \mathrm{~m}\right)$ and a 1-shell collision object $\left(m_{c}=4.5 \mathrm{~kg}, r_{c}=0.1 \mathrm{~m}\right.$ at $v_{c}=0.5 \mathrm{~m} / \mathrm{s}^{3}$ The task of the robot is to hold $q_{d}=0 \mathrm{~m}$ at $K_{d}^{*}=5000 \mathrm{~N} / \mathrm{m}$. Please note that for this one-dimensional collision $q$ denotes the Cartesian position. The influence of the respective key parameters is investigated regarding the equilibrium, stiffness, and feedforward torque adaption.

Figure 7 depicts the $\boldsymbol{q}_{d}$-adaptation during a single collision. The top chart shows the stress spiking $p(\sigma)$ evoked by the

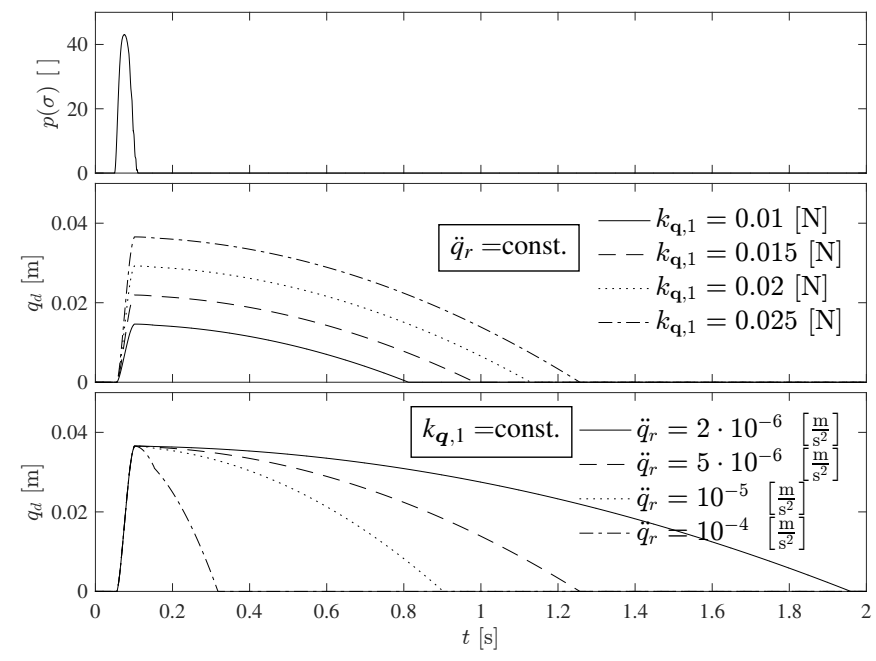

Fig. 7. $\boldsymbol{q}_{d}$-adaptation for single contact for different $\boldsymbol{k}_{\boldsymbol{q}}$ and $\ddot{q}_{r}$.

collision. In the middle plot, $\boldsymbol{q}_{d}$-adaptation based on $p(\sigma)$ and in dependence of $k_{q, 1}$ from the gain vector $k_{q}$ is shown at fixed recovery acceleration $\ddot{q}_{r}$. Obviously, $k_{\boldsymbol{q}, 1}$ can be used for varying the magnitude of the set point. The lower chart shows the reverse case at fixed value of $k_{\boldsymbol{q}, 1}$. This means that for a given collision the distal response does not change, while the recovery acceleration $\ddot{q}_{r}$ varies, allowing to influence the decay time before the robot proceeds with the task. In Fig. 8 the variation of stiffness rate adaptation is shown during repetitive contacts. The top diagram depicts the

\footnotetext{
${ }^{3}$ The radii $r_{r, c}$ are equally chosen, since the used contact model is only valid for radii greater than the contact radius $d$, see Fig. 3. Reasonable values are chosen for $m_{r, c}$ that correspond to typically reflected mass values found during human-robot collisions [15].
} 
TABLE II

GAIN VECTORS AND PARAMETERS FOR COLLISION BETWEEN BIOTAC AND HUMAN FINGER (SEE FIG. 10).

\begin{tabular}{|c|c|c|c|c|c|}
\hline \multirow{2}{*}{ pain class } & \multicolumn{2}{|c|}{$\boldsymbol{q}_{d}$-adapt. } & \multicolumn{3}{|c|}{$\boldsymbol{K}_{d}$-adapt. } \\
\hline & $\boldsymbol{k}_{\boldsymbol{q}}^{T}[\mathrm{~N}--]$ & $\ddot{\boldsymbol{q}}_{r}\left[\frac{\mathrm{rad}}{\mathrm{s}^{2}}\right]$ & $\boldsymbol{k}_{K}^{T}\left[--\frac{\mathrm{Nm}}{\mathrm{rad} \cdot \mathrm{s}}\right]$ & $\boldsymbol{K}_{d}^{*}\left[\frac{\mathrm{Nm}}{\mathrm{rad}}\right]$ & $\ddot{\boldsymbol{K}}_{r}\left[\frac{\mathrm{Nm}}{\mathrm{rad} \cdot \mathrm{s}^{2}}\right]$ \\
\hline I & $\mathbf{0}^{T}$ & 0 & $\mathbf{0}^{T}$ & $\operatorname{diag}\{500\}$ & 0 \\
\hline II & {$\left[\begin{array}{lll}0.05 & 0 & 0\end{array}\right]^{T}$} & $\operatorname{diag}\left\{10^{-5}\right\}$ & {$\left[\begin{array}{lll}0 & 0 & 60\end{array}\right]^{T}$} & $\operatorname{diag}\{500\}$ & $\operatorname{diag}\{0.02\}$ \\
\hline III & {$\left[\begin{array}{llll}0.075 & 0 & 0\end{array}\right]^{T}$} & $\operatorname{diag}\left\{5 \cdot 10^{-6}\right\}$ & {$\left[\begin{array}{lll}0 & 0 & 80\end{array}\right]^{T}$} & $\operatorname{diag}\{500\}$ & $\operatorname{diag}\{0.02\}$ \\
\hline
\end{tabular}

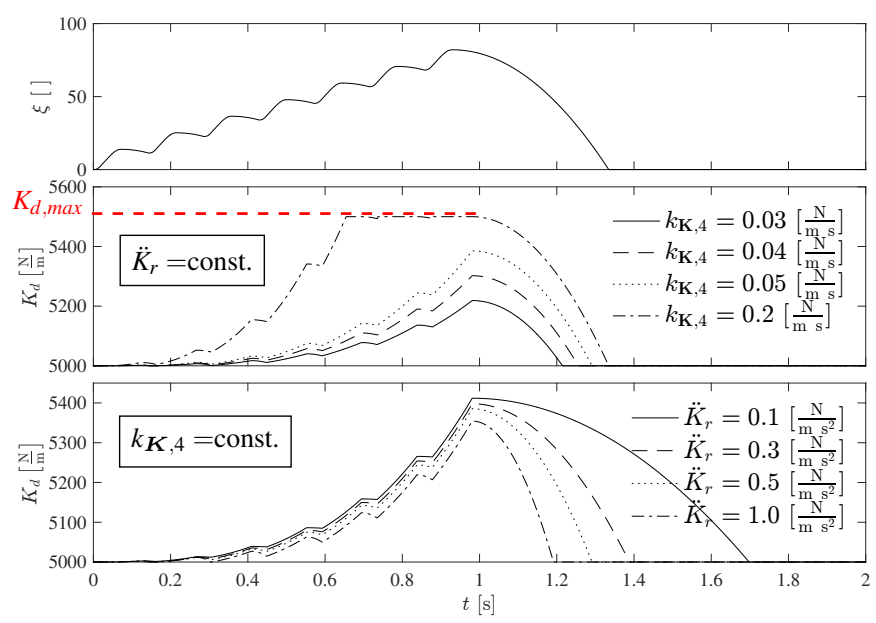

Fig. 8. $\boldsymbol{K}_{d}$-adaptation for repetitive contacts for different $\boldsymbol{k}_{K}$ and $\ddot{K}_{r}$.

repetitiveness spiking $\xi$. The middle and lower diagrams show $\boldsymbol{K}_{d}$-adaptation for different $k_{K, 4}$ at fixed recovery acceleration $\ddot{K}_{r}=$ const. (middle) and vice versa (bottom). The stiffness magnitude increases with the number of contacts (indicated by $\xi$ ) at different rates depending on the particular choice of $\boldsymbol{k}_{\boldsymbol{K}}$ until $K_{p, \max }=5500 \mathrm{~N} / \mathrm{m}$ is reached. When the contact event is over $\boldsymbol{K}_{d}$ recovers to the nominal stiffness $K_{d}^{*}$ with $\ddot{K}_{r}$. Figure 9 shows feedforward torque adaption for $r_{\max }(\delta)=16$, $r_{v, \max }(\boldsymbol{\delta})=2200 \mathrm{~s}^{-1}$, and $f_{p, \max }=100 \mathrm{~N}$. The first and

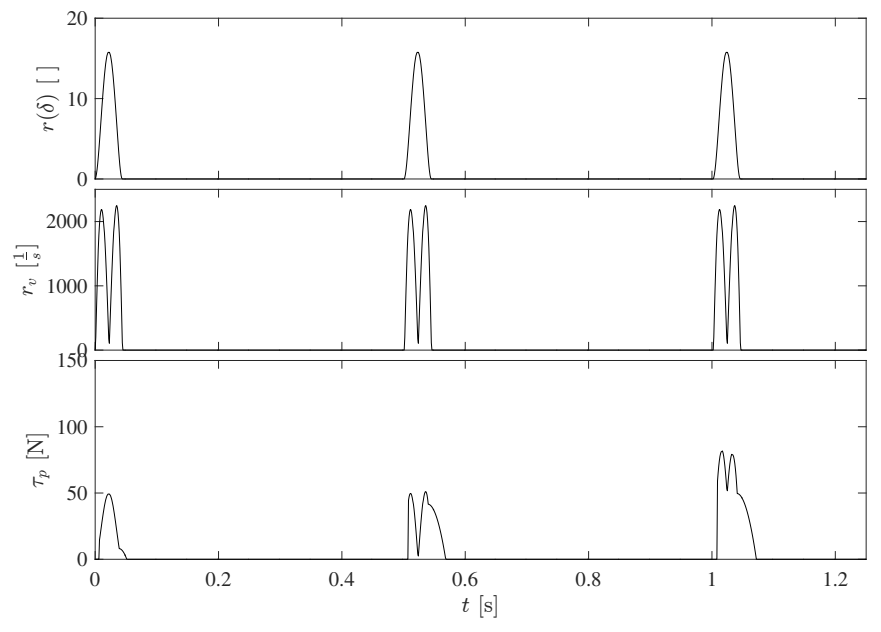

Fig. 9. Contribution of $r(\delta)$ and $r_{v}$ to $\boldsymbol{\tau}_{p}$-adaptation for given $\boldsymbol{k}_{\boldsymbol{\tau}}$.

middle chart depict the penetration depth and velocity spiking $r(\boldsymbol{\delta})$ and $r_{v}$ for three consecutive collisions. To separately emphasize the contribution of the two spiking signals only $r(\boldsymbol{\delta})$ is activated for the first collision. During the second collision, only $r_{v}$ is considered, while the last collision shows the combined response. The decay acceleration is set to $\ddot{\tau}_{r}=50 \mathrm{~N} / \mathrm{s}^{2}$.

To sum up, the aRNS controller adaption can be separately set up for $\boldsymbol{q}_{s}, \boldsymbol{K}_{d^{-}}$, and $\boldsymbol{\tau}_{f f}$, which should be done carefully, while considering the used robot, its task, and foreseeable environments.

\section{B. Pain reaction}

The pain-reflex control is experimentally demonstrated using a KUKA LWR4+ equipped with the BioTac ${ }^{\circledR}$ sensor as an implementation of the artificial robot neuron concept. The sensor is mounted at the end effector and pressure is induced by a human finger, leading to the respective pain class and reflex reaction, see Fig. 10. The BioTac can sense multiple modalities such as pressure, vibration, temperature or spatial contact forces. In this paper, we utilize, e.g., the pressure signal $\sigma_{B T}$ as the cumulated spiking signal adapting $\boldsymbol{q}_{d}$. The

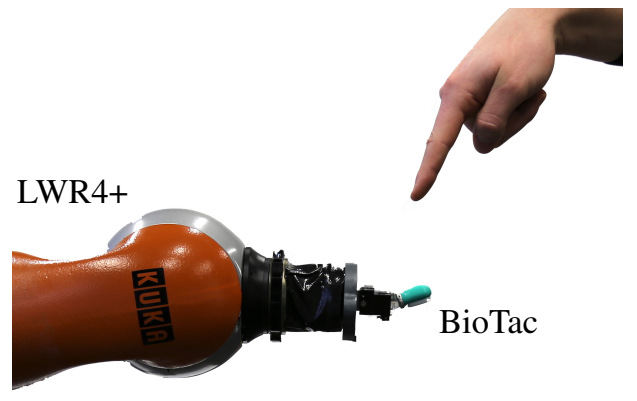

Fig. 10. Experimental setup consisting of KUKA LWR4+ equipped with BioTac sensor and controlled by the aRNS.

repetitiveness spiking $\xi$ is realized by a counter with constant gradient and exponential decay characteristics that is activated for $\sigma_{B T}>0$, directly influencing $\boldsymbol{K}_{d}$. The particular relation can be derived from (8) by replacing $a F \delta$ with $T_{i n c}=0.1$ and $\dot{\delta}$ with $\sigma_{B T}$. In addition, we extend the spiking state vector $s$ in (14) by the modality of temperature sensing, utilizing the analog derivative of temperature $T_{A C}$ of the BioTac. ${ }^{4}$ Due to the larger time constants of temperature measurement we introduce a separate temperature pain class that is activated as soon as a threshold of $H=1920$ kbits is exceeded. Its formal definition and reflex behavior is equivalent to the other contact classes. However, only two reaction classes are defined for this

\footnotetext{
${ }^{4}$ For more information about the BioTac and signal interpretation see [21].
} 
particular case. The robot retracts with a change of $5 \mathrm{deg}$ in every joint and waits $4 \mathrm{~s}$ until recovery, provided that $T_{A C}$ does not drop any further. These values were empirically found to successfully avoid contact with a plastic cup filled with hot boiled water $\left(\vartheta_{c} \approx 100^{\circ} \mathrm{C}\right)$.

During the experiment the cup was only slightly touched in order to avoid unwanted retraction due to pressure sensing. The spiking state vector used in the experiment is $\boldsymbol{s}_{B T}=$ $\left[\sigma_{B T}, T_{A C}, \xi\right]^{T}$ and the controller parameters can be found in Tab. II. The equilibrium position related parameters are empirically chosen such that for the given contact events the robot does not violate a predefined workspace, while the stiffness parameters comply with the specifications of the KUKA LWR4+. The robot task is to mantain $\boldsymbol{q}_{d}=$ $[0,30,0,-50,0,10,0]^{T}$ [deg]. In Figure 11 one can see an increase in desired equilibrium position and a slight increase in stiffness for light pain. For moderate pain one observes a reinforced version of light pain. Note that since the relevant reflex behavior in this particular experimental setup was mainly observed in axis 2 and 4, we omit the other traces for sake of clarity. The corresponding gains were selected to be larger and the recovery rates decreased. The collision that causes severe pain represents a special case where the controller switches to pure gravity compensation mode with some additional damping, see Fig. 6. The pain-controls are deactivated and without human intervention the robot is not able to return to the task. Most noticeably, the set point adaption and stiffness rise until contact is lost during repetitive contacts. Finally, the robot retracts to avoid longer contact with a hot object for the temperature reflexes.

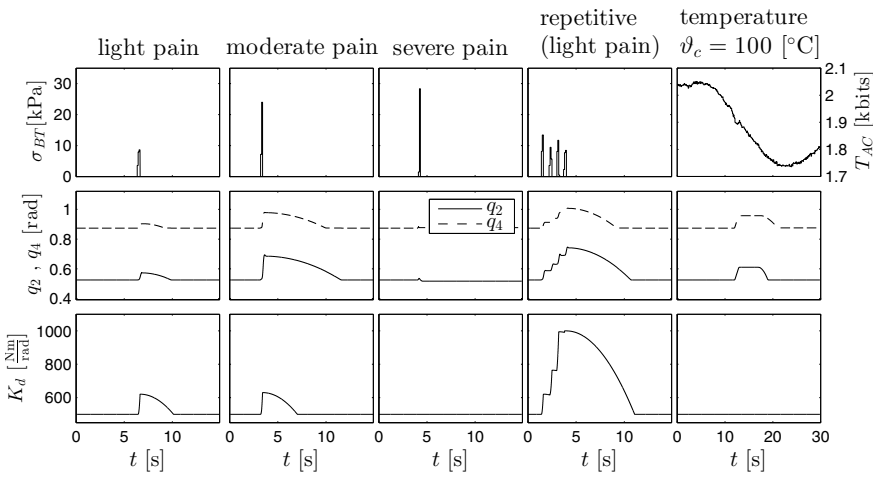

Fig. 11. Physical collision for the KUKA LWR4+ equipped with the aRNS/BioTac and a human finger that applies varying pressure.

\section{CONCLUSION}

This paper is our first step towards enabling robots to not only measure or estimate contacts, but also to sense and interpret them based on novel bio-inspired controls. For this, we developed the concept of an artificial Robot Nervous System (aRNS), which architecture and basic functionality mimics its human antetype. The concept is designed to unify different sensing modalities and let the robot respond in a humaninspired way to perceived stimuli. Specifically, we introduce a sensory pathway from mechanical collision quantities such as contact forces and stresses to arificial Robot Neuron firing caused by quantities such as penetration depth, contact timing, and aRN density in the involved tissue layers. This concept makes it possible to design new and interesting collision reflex reaction behaviors. Depending on spiking rate the robot executes protective behavior of varying intensity as an escape strategy before trying to re-engage into the previous task again.

\section{REFERENCES}

[1] M. Zinn, O. Khatib, B. Roth, and J. Salisbury, "Playing it safe - humanfriendly robots," IEEE Robotics and Automation Mag., vol. 11, pp. 12 21, 2002.

[2] S. Haddadin, A. Albu-Schäffer, and G. Hirzinger, "Safety evaluation of physical human-robot interaction via crash-testing," in Robotics: Science and Systems, vol. 3, 2007, pp. 217-224.

[3] M. A. P. Mark F. Bear, Barry W. Conners, Neuroscience - Exploring the Brain. Lippincott Williams \& Wilkins, 2007.

[4] Q. H. Q. Huang and Y. Nakamura, "Sensory reflex control for humanoid walking," IEEE Trans. on Robotics, vol. 21, no. 5, pp. 977-984, 2005.

[5] R. Kratz, S. Klug, M. Stelzer, and O. von Stryk, "Biologically inspired reflex based stabilization control of a humanoid robot with artificial sma muscles," in EEE Int. Conf. on Robotics and Biomimetics, 2006, pp. 1089-1094.

[6] S. Yakovenko, V. Gritsenko, and A. Prochazka, "Contribution of stretch reflexes to locomotor control: a modeling study," Biological Cybernetics, vol. 90 , no. 2 , pp. 146-155, 2004.

[7] M. M. Williamson, "Postural primitives: Interactive behavior for a humanoid robot arm," Society for Adaptive Behaviour, 1996.

[8] S. Yigit, C. Burghart, and H. Woern, "Applying reflexes to enhance safe human-robot-co-operation with humanlike robot arm," Int. Symp. on Robotics, pp. 1-6, 2004.

[9] T. S. Dahl and A. Paraschos, "A force-distance model of humanoid arm withdrawal reflexes," in FIRA-TAROS, 2012, pp. 13-24.

[10] S. Haddadin, Towards Safe Robots, ser. Springer Tracts in Advanced Robotics. Springer Berlin Heidelberg, 2014, no. 90.

[11] A. De Luca, A. Albu-Schäffer, S. Haddadin, and G. Hirzinger, "Collision, detection and safe reaction with the dlr-iii lightweight manipulator arm," in Int. Conf. on Intelligent Robots and Systems, 2006 IEEE/RSJ. IEEE, 2006, pp. 1623-1630.

[12] S. Haddadin, A. Albu-Schäffer, A. De Luca, and G. Hirzinger, "Collision detection and reaction: A contribution to safe physical human-robot interaction," in Int. Conf. on Intelligent Robots and Systems, 2008. IEEE/RSJ. IEEE, 2008, pp. 3356-3363.

[13] S. Haddadin, F. Huber, and A. Albu-Schäffer, "Optimal control for exploiting the natural dynamics of variable stiffness robots," in IEEE Int. Conf. on Robotics and Automation. IEEE, 2012, pp. 3347-3354.

[14] S. Ivaldi, "From humans to hhumanoid: a study on optimal motor control for the icub," Ph.D. dissertation, Italian Institute of Technology \& University of Genova, Italy, 2011.

[15] J.-J. Park, S. Haddadin, J.-B. Song, and A. Albu-Schäffer, "Designing optimally safe robot surface properties for minimizing the stress characteristics of human-robot collisions," in IEEE Int. Conf. on Robotics and Automation, 2011, pp. 5413-5420.

[16] R. S. Johansson and A. B. Vallbo, "Tactile sensibility in the human hand: Relative and absolute densities of four types of mechanoreceptive units in glabrous skin," Journal of Physiology, vol. 286, pp. 283-300, 1979.

[17] R. Polianskis, T. Graven-Nielsen, and L. Arendt-Nielsen, "Pressure-pain function in desensitized and hypersensitized muscle and skin assessed by cuff algometry," The Journal of Pain, vol. 3, no. 1, pp. 28-37, February 2002.

[18] H. Fruhstorfer, T. Mueller, and E. Scheer, "Capillary blood sampling: how much pain is necessary? part2: Relation between penetration depth and puncture pain," Practical Diabetes International, vol. 12, no. 4, pp. 184-185, July/August 1995.

[19] M. Hollins, D. Harper, and W. Maixner, "Changes in pain from a repetitive thermal stimulus: The roles of adaptation and sensitization," Pain, vol. 152, no. 7, pp. 1583-1590, March 2011.

[20] A. Albu-Schaffer, C. Ott, U. Frese, and G. Hirzinger, "Cartesian impedance control of redundant robots: recent results with the dlr-lightweight-arms," in Robotics and Automation, 2003. Proceedings. ICRA '03. IEEE International Conference on, vol. 3, Sept 2003, pp. 37043709 vol.3.

[21] J. A. F. Danfei Xu, Gerald E. Loeb, "Tactile identification of objects using bayesian exploration," in International Conference on Robotics and Automation (ICRA), 2013. 